\title{
Statin use and exacerbations in individuals with chronic obstructive pulmonary disease
}

\author{
Truls S Ingebrigtsen, 1,2,3 Jacob L Marott, ${ }^{2}$ Børge G Nordestgaard, ${ }^{2,3,4}$ \\ Peter Lange, ${ }^{2,3,5,6}$ Jesper Hallas, ${ }^{7}$ Jørgen Vestbo ${ }^{8,9}$
}

- Additional material is published online only. To view these images and file please visit the journal online (http:// dx.doi.org/10.1136/thoraxjnl2014-205795)

For numbered affiliations see end of article.

\section{Correspondence to}

Professor Jørgen Vestbo, Department of Respiratory Medicine, Gentofte Hospital, Niels Andersens Vej, 65, Hellerup 2900, Denmark; Jorgen.Vestbo@manchester. ac.uk

Received 29 May 2014 Revised 18 August 2014 Accepted 1 October 2014 Published Online First 27 October 2014

\begin{abstract}
Background We tested the hypothesis that statin use in individuals with COPD is associated with a reduced risk of exacerbations.

Methods We identified 5794 individuals with COPD and a measurement of $C$ reactive protein (CRP) in the Copenhagen General Population Study (2003-2008). During 3 years of follow-up we recorded exacerbations with hospital admissions or oral corticosteroid treatment. In a nested case-control design, matching on age, gender, smoking, COPD severity and comorbidity, we estimated the association between statin use and exacerbations. In addition, we examined the association between statin use and high CRP (>3 mg/L), and the association between high CRP and exacerbations during follow-up.
\end{abstract}

Results Statin use was associated with reduced odds of exacerbations in crude analysis, $\mathrm{OR}=0.68(95 \% \mathrm{Cl} 0.51$ to $0.91, p=0.01$ ), as well as in multivariable conditional logistic regression analysis, $\mathrm{OR}=0.67$ ( 0.48 to 0.92 , $\mathrm{p}=0.01$ ). However, in the subgroup with the most severe COPD and without cardiovascular comorbidity, we observed a null association between statin use and exacerbations, $O R=1.1$ ( 0.5 to $2.1, p=0.83$ ).

Furthermore, statin use was associated with reduced odds of a high CRP, OR=0.69 (0.56 to $0.85, p<0.001)$, and a high CRP was associated with an increased risk of exacerbations, $H R=1.62$ (1.35 to $1.94, p<0.001)$. We estimated the percentage of excess risk of the association of statin use with exacerbations possibly mediated through a reduction of CRP to be $14 \%$ (4-51\%).

Conclusions Statin use was associated with reduced odds of exacerbations in individuals with COPD from the general population, although this was not apparent in those with the most severe COPD without cardiovascular comorbidity. Statins may thus only associate with reduced risk of exacerbations in patients with COPD with coexisting cardiovascular disease.

\section{INTRODUCTION}

COPD is one of the most important global health problems. ${ }^{1} 2$ COPD is characterised by exacerbations, and these exacerbations constitute key events in COPD progression, prognosis and treatment. ${ }^{34}$

In recent years there has been a growing body of evidence suggesting systemic inflammation as a key element in the pathogenesis of COPD. ${ }^{5}{ }^{6}$ As a result, there is an increasing focus on whether use of medications that reduce markers of systemic inflammation may also reduce the risk of exacerbations in COPD. ${ }^{7}$

\section{Key messages}

What is the key question?

- Do statins have beneficial effects on exacerbations in unselected individuals with COPD, and if so, what might be part of the explanation?

What is the bottom line?

- Several population based studies have indicated that statin use may reduce the risk of exacerbations in unselected individuals with COPD, but studies have lacked clinical characteristics such as severity of COPD, comorbidities and markers of systemic inflammation.

\section{Why read on?}

- In unselected individuals from the general population our study indicates that statin use is associated with a reduction in exacerbations in COPD, but like in a recent randomised controlled trial we observed a null association in those with the most severe COPD and without cardiovascular comorbidity suggesting that statins may only associate with reduced risk of exacerbations in patients with COPD with coexisting cardiovascular disease.

Statins have effects besides a lowering of plasma cholesterol, including anti-inflammatory effects, which have been investigated in several studies. ${ }^{8} 9$ Studies have also shown that statin use has beneficial effects on cardiovascular outcomes, possibly by reduction of $\mathrm{C}$ reactive protein (CRP) levels. ${ }^{10} 11$

In COPD, pharmaceutical database studies have indicated a possible beneficial effect of statin use on exacerbations. ${ }^{12}$ Although this evidence comes from several populations, studies have lacked clinical characteristics and data on markers of systemic inflammation. ${ }^{7}{ }^{13}$ Furthermore, a recent large randomised trial found no effect of simvastatin on exacerbations in patients with the most severe COPD, but without cardiovascular comorbidity. $^{14}$

We tested the hypothesis that statin use in individuals with COPD is associated with reduced risk of exacerbations. This hypothesis was tested among individuals with different severity of COPD and cardiovascular comorbidity, as well as in the subgroup with the most severe COPD and without cardiovascular comorbidity. 


\section{METHODS}

\section{Population}

We used data from the Copenhagen General Population Study (2003-2008) comprising 55731 participants. For this study, we identified 5812 individuals with COPD, defined by presence of airflow limitation $\left(\mathrm{FEV}_{1}\right.$ divided by $\left.\mathrm{FVC}, \mathrm{FEV}_{1} / \mathrm{FVC}<0.7\right)$, no self-reported asthma and age above 40 years. $^{15} 16$ Of these, $5794(99.7 \%)$ individuals had measurement of CRP at the examination.

\section{Linkage to national all-inclusive registers}

From the all-inclusive Danish National Patient Registry ${ }^{17}$ we retrieved data on hospital admissions with a discharge diagnosis of COPD (ICD-10: J41-J44) to define severe exacerbations of COPD. ${ }^{15}{ }^{18}$ In addition, we identified previous hospital admissions with a discharge diagnosis of ischaemic heart disease, and/ or diabetes mellitus as part of the comorbidity definition; see online supplementary e-table 1 for further details.

From the Danish National Prescription Registry, ${ }^{19}$ we retrieved data on treatment with oral corticosteroids alone or in combination with antibiotics to define moderate exacerbations of COPD. ${ }^{15}{ }^{18}$ Furthermore, we included data on previous use and dose of maintenance pulmonary medications (long-acting bronchodilators and fixed-dose combinations with inhaled corticosteroids) in either the 3 months before an exacerbation, or in the 3 months before measurement of CRP at the examination. In addition, we assessed the use and dose of statins during either the 3 months before an exacerbation, or the 3 months before measurement of CRP. We had access to dispensed medication data from 1995 until 31 December 2009, thus ensuring at least 1 year of complete follow-up for all individuals. See online supplementary e-table 2 for all study anatomical therapeutical chemical $\operatorname{codes}^{20}$ used to identify dispensed medications.

\section{Study approval}

The study was approved by an institutional review board and the regional ethics committee (H-KF01-144/01), and was conducted according to the Declaration of Helsinki. Written informed consent was obtained from all participants.

\section{Study design}

Figure 1 shows the study design.

\section{Statin use and exacerbations}

To examine the association between statin use and odds of exacerbations, we applied a nested case-control design; see flow diagram in online supplementary e-figure $1 .^{12}$ Cases were individuals with a first exacerbation within a maximum of 3 years of follow-up. Exacerbations were defined as a composite of medically treated exacerbations and hospital admissions with COPD. ${ }^{18}$ Statin use was defined as at least one dispense of statins in the 3 months prior to the exacerbation date for cases. The exacerbation date for cases defined an index date. Controls were assigned the same index date as their corresponding case, and statin use was calculated in the 3 months prior to this index date. Controls were selected from the COPD cohort by use of risk-set sampling technique. ${ }^{21}$ Individuals eligible to become controls were those who matched the index case with respect to age, gender, smoking, COPD severity (Global Initiative for Chronic Obstructive Lung Disease, GOLD grade) and cardiovascular comorbidity, and who did not have an exacerbation prior to the index date (see online supplementary e-table 1 for further details), ${ }^{42}$ but controls could later become a case, thus ensuring that the ORs are unbiased estimates of the incidence rate ratio. $^{21}$ Two controls were selected for each case. The reason for choosing a nested case-control design was that these casecontrol studies are computationally more effective than cohort studies, while producing the same estimates as a cohort study with little loss in precision. ${ }^{23-25}$

In addition, we selected a subgroup of individuals with COPD who were $40-80$ years of age, had $\mathrm{FEV}_{1}<80 \%$, previous exacerbations (within 10 years), and without cardiovascular comorbidity. This was done to mimic the patients enrolled in a recent randomised controlled trial (RCT). ${ }^{14}$ Among these individuals, we identified 89 cases with exacerbations, and selected
Figure 1 Study design. 'Figure 2': Conditional logistic regression analysis in case-control study of statin use and associated odds of exacerbations. 'Figure 3': Logistic regression analysis of statin use and associated odds of having a high $C$ reactive protein (CRP) level (>3 mg/L). 'Figure 4': Cox regression analysis of high CRP and associated risk of exacerbations. 'Figure 5': Estimated percentage of excess risk of the association of statin use with exacerbations possibly mediated through CRP, percentage of excess risk mediated (PERM) (CRP).
Study design

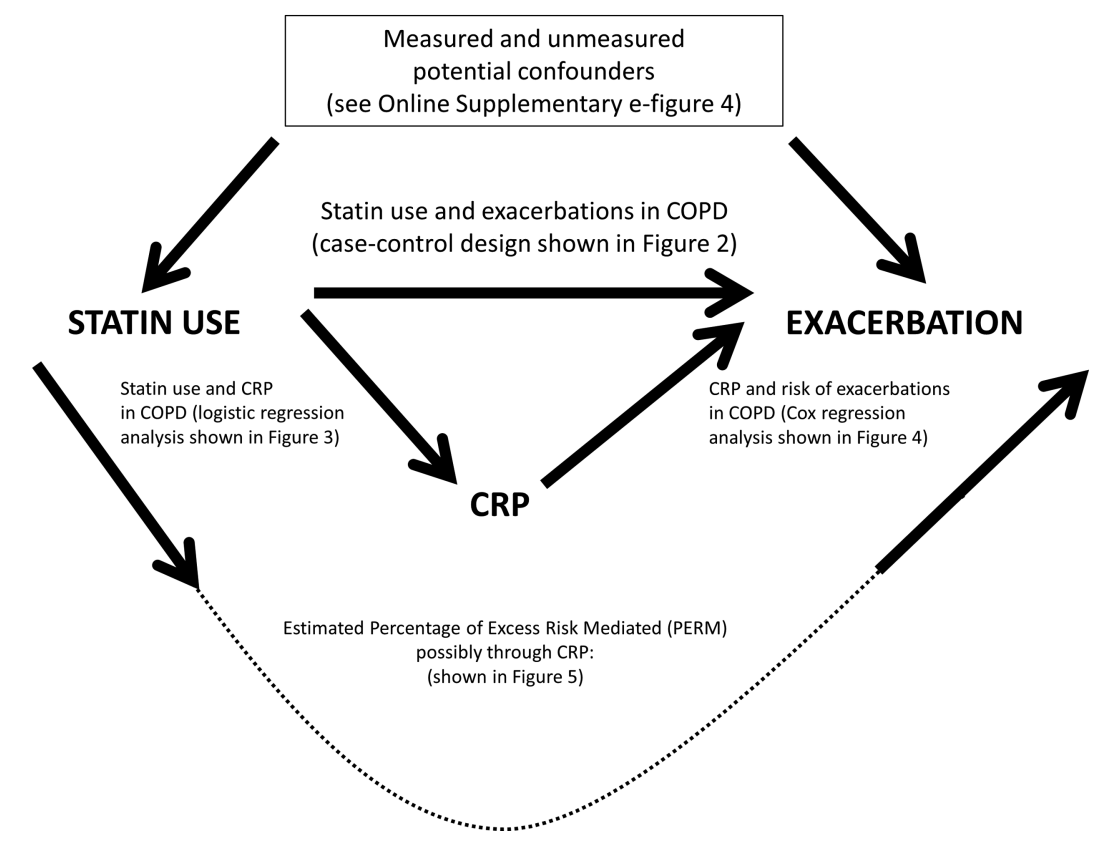


168 controls as described above, except that the subgroup selection criteria only allowed matching on age, gender and smoking.

\section{Statin use and CRP}

We assessed the association between statin use and CRP (see figure 1) by examining the relationship between statin use in the 3 months before the measurement of CRP at the examination, and as main outcome a categorised CRP (low $\leq 3 \mathrm{mg} / \mathrm{L}$ <high). ${ }^{18}$

\section{CRP and exacerbations}

We analysed the association between a high CRP at the examination and the risk of exacerbations within 3 years of follow-up (see figure 1), by analysing the time to first exacerbation.

\section{Percentage of excess risk mediated}

We also estimated the percentage of excess risk of the association of statin use with exacerbations in COPD possibly mediated through CRP, percentage of excess risk mediated (PERM) (CRP). ${ }^{26}$

\section{Statistical analyses Demographics}

The statistical software package $\mathrm{R}$ (V.3.0.1) was used. ${ }^{27}$ Characteristics of individuals with COPD who were statin users versus those who did not use statins were evaluated using $\chi^{2}$ tests or ANOVA for categorical or continuous variables as appropriate. $^{28}$

\section{Statin use and exacerbations}

We performed a conditional logistic regression analysis (R-package $=$ 'survival'), to assess the association between statin use in the 3 months before an exacerbation and odds of exacerbations, in the case-control design. ${ }^{12}$ To assess a possible dose-response relationship, we also included dose of statins (low dose $\leq 50 \%$ of defined daily dosages $<$ high dose). ${ }^{12}{ }^{20}$ We then adjusted the conditional logistic regression analysis for the level of CRP (high/low). ${ }^{18}$ Furthermore, we applied a multivariable conditional logistic regression analyses where we included as confounders the use of all maintenance pulmonary medications, health behaviour (regular use of vitamin pills and regular visits to the general practitioner), dose of all maintenance pulmonary medications (high/low) and CRP level (high/low). See online supplementary e-table 1 for a detailed description of all variables.

In sensitivity analyses, we also adjusted our estimates for $\mathrm{FEV}_{1}$. This was done to see if inclusion of exact pulmonary function, in addition to matching on the rather broad GOLD grades, would affect the size of our estimates. Furthermore, we tested if socioeconomic status assessed by education or household income, ${ }^{29}$ alcohol consumption categories (daily/weekly or monthly/none) $)^{30}$ and frailty assessed by reporting the feeling of not having accomplished very much recently or reporting a feeling of giving up, were associated with exacerbations or affected our estimates. ${ }^{31}$

Individuals with missing values on confounders in multivariable models were not included in the analysis, explaining why the numbers reported in the multivariable models differ slightly. We had complete register follow-up data on all individuals. The average time until first exacerbation was 452 days.

In the subgroup analysis among those with the most severe COPD and without cardiovascular comorbidity, we performed the same analyses as described in our main analysis above.
Statin use and CRP

The association between statin use during the 3 months prior to measurement of CRP at the examination, and high or low CRP was analysed in a logistic regression analysis. ${ }^{18}$ We adjusted our analyses for the matching variables included in the case-control design described above: age, gender, GOLD grade, smoking and comorbidity. We then included the confounders of use of all maintenance pulmonary medications, health behaviour and doses of all maintenance pulmonary medications (see online supplementary e-table 1 for details). In a sensitivity analysis, we included $\mathrm{FEV}_{1}$ as a possible confounder.

Using the multivariable logistic regression model, ${ }^{28}$ we calculated the average predicted probability of having a high CRP in the group using statins and in the group not using statins.

\section{CRP and exacerbations}

We analysed time to first exacerbation using Cox regression analysis. Censoring was death, emigration or end of follow-up. First, we adjusted our analyses for age, gender, GOLD grade, smoking and comorbidity. In a sensitivity analysis, we included an additional CRP cut point of $1 \mathrm{mg} / \mathrm{L}$ in the analysis (see online supplementary e-table 1 for details). Then we included the confounders of use of all maintenance pulmonary medications, health behaviour, dose of all maintenance pulmonary medications, and in a sensitivity analysis $\mathrm{FEV}_{1}$.

Using the multivariable Cox regression model, ${ }^{28}$ we calculated the average predicted probability of exacerbations in the group with high CRP and in the group with low CRP.

Furthermore, in a sensitivity analysis to see if high CRP is a stable predictor of exacerbations over time, we also started follow-up from 6 months after the measurement of CRP, and followed our participants for a maximum of 3 years.

\section{Percentage of excess risk mediated}

We estimated the percentage of excess risk of the association of statin use with exacerbations in COPD possibly mediated by CRP, PERM(CRP), ${ }^{26}$ as,

$$
\begin{aligned}
& P E R M(C R P)= \\
& \frac{R R(\text { confounder adjusted })-R R(\text { confounder and CRP adjusted })}{R R(\text { confounder adjusted })-1}
\end{aligned}
$$

CIs were estimated by bootstrap resampling (10 000 samples). ${ }^{32}$ As a sensitivity analysis we estimated PERM(CRP) by including $\mathrm{CRP}$ as a continuous variable instead of a categorical variable (high/low).

\section{RESULTS}

\section{Demographics}

Among 5794 individuals with COPD, we identified 700 (12.1\%) individuals who were statin users in the 3 months before the examination date in the Copenhagen General Population Study. Statin users were older than non-users (71 vs 66 years, $\mathrm{p}<0.001)$, were more likely to be male $(53 \%$ vs $47 \%$, $\mathrm{p}<0.001)$ and they were less likely to be current smokers $(31 \%$ vs $40 \%, \mathrm{p}<0.001)$. As expected, statin users were more likely to have cardiovascular comorbidities than non-users $(63 \%$ vs $18 \%$, $\mathrm{p}<0.001$ ) and they were more likely to have visited their general practitioner regularly (more than three times in the previous year, $55 \%$ vs $29 \%, \mathrm{p}<0.001)$. Statin users did not differ from non-users with respect to $\mathrm{FEV}_{1}$ or use of maintenance pulmonary medications; further details are shown in the left part of online supplementary e-table 3 . 
During the 3 year follow-up we identified 530 cases with exacerbations, and selected 1016 corresponding controls. There were no statistically significant differences between cases and controls for any of the matching variables (all $\mathrm{p}$ values $>0.10$, $\chi^{2}$ tests). Among 1546 individuals with COPD in the casecontrol study, we identified $320(20.7 \%)$ who were statin users during the 3 months before the index date. The right part of online supplementary e-table 3 shows characteristics of those individuals with statin use versus those not using statins in the case-control study.

\section{Statin use and exacerbations}

Statin use in the 3 months before the index date was associated with reduced odds of exacerbations in crude conditional logistic regression analysis, $\mathrm{OR}=0.68$ (95\% CI 0.51 to $0.91, \mathrm{p}=0.01)$, as well as in multivariable conditional logistic regression analysis, $\mathrm{OR}=0.67(0.48$ to $0.92, \mathrm{p}=0.01)$ compared with no use of statins. Estimates were generally robust towards possible confounder adjustments, as shown in the left part of figure 2 .

In sensitivity analyses, only $\mathrm{FEV}_{1}$ and education contributed significantly. When including $\mathrm{FEV}_{1}$ in the multivariable model the association of statin use with exacerbations remained similar, as shown in figure 2, and this also applied when including education $(\mathrm{OR}=0.68$ (0.5 to 1.0$), \mathrm{p}=0.049$, for the association between statin use and exacerbations).

The online supplementary e-figure 2 shows a forest plot of possible confounding variables included in multivariable conditional logistic regression analysis and their corresponding odds of exacerbations.
In the subgroup analysis among 257 individuals (89 cases and 168 controls) with the most severe COPD and without cardiovascular comorbidity, we observed a null association between statin use and exacerbations, $\mathrm{OR}=1.3(0.7$ to $2.4, \mathrm{p}=0.46)$ in crude conditional logistic regression analysis, and $\mathrm{OR}=1.1(0.5$ to $2.1, \mathrm{p}=0.83$ ) in multivariable conditional logistic regression analysis, as shown in the right part of figure 2 .

\section{Statin use and CRP}

As shown in figure 3, statin use in the 3 months before measurement of CRP at the examination reduced the odds of having a high CRP ( $>3 \mathrm{mg} / \mathrm{L}), \mathrm{OR}=0.69$ (0.56 to $0.85, \mathrm{p}<0.001)$, in multivariable logistic regression analysis. The average predicted probability of having a high CRP in the group using statins was $23 \%$ compared with $30 \%$ in the group not using statins (figure 3). The online supplementary e-figure 3 shows a forest plot of possible confounding variables included in multivariable logistic regression analysis and the corresponding odds of having a high CRP.

\section{CRP and exacerbations}

Multivariable Cox regression analysis showed that having a high CRP in COPD was associated with an increased risk of exacerbations during follow-up, $\mathrm{HR}=1.62$ (1.35 to $1.94, \mathrm{p}<0.001$ ), as shown in figure 4 . The average predicted probability of having exacerbations during follow-up was $13 \%$ in the group with high CRP compared with $8 \%$ in the group with low CRP (figure 4).
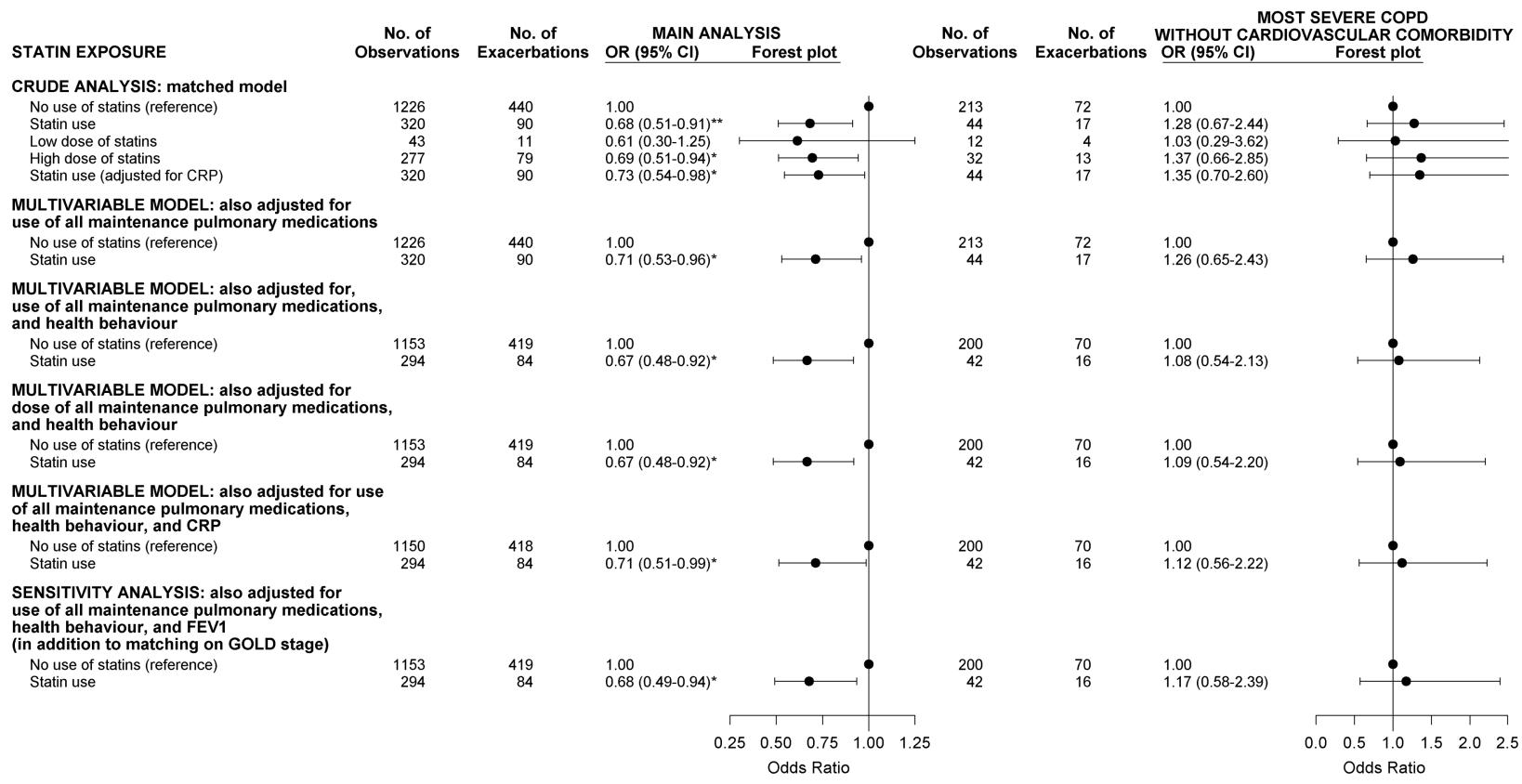

Figure 2 Statin use and exacerbations. Results of the crude and multivariable conditional logistic regression analyses. Left part: Main analysis. Case-control study among 1546 individuals with COPD from the general population. From left to right: number of individuals in study ('No. of Observations'), number of individuals with exacerbations ('No. of Exacerbations'), OR of exacerbation with 95\% Cls, and a corresponding forest plot. Significance codes: ${ }^{* * \prime}$ for $p<0.01 ;{ }^{\prime * \prime}$ for $p<0.05$. Ninety-nine individuals did not have data on health behaviour, and an additional three individuals did not have data on $C$ reactive protein (CRP), explaining why the number of observations differ between the multivariable models. CRUDE ANALYSIS: matched on age, gender, smoking, COPD severity (Global Initiative for Chronic Obstructive Lung Disease, GOLD grade), and cardiovascular comorbitidy. Right part: Most severe COPD without cardiovascular comorbidity. Subgroup analysis among 257 individuals with the most severe COPD and without cardiovascular comorbidity. From left to right: number of individuals in study ('No. of Observations'), number of individuals with exacerbations ('No. of Exacerbations'), OR of exacerbation with 95\% Cls, and a corresponding forest plot. Fifteen individuals did not have data on health behaviour explaining why the number of observations differ between the multivariable models. CRUDE ANALYSIS for this subgroup: matched on age, gender and smoking. 


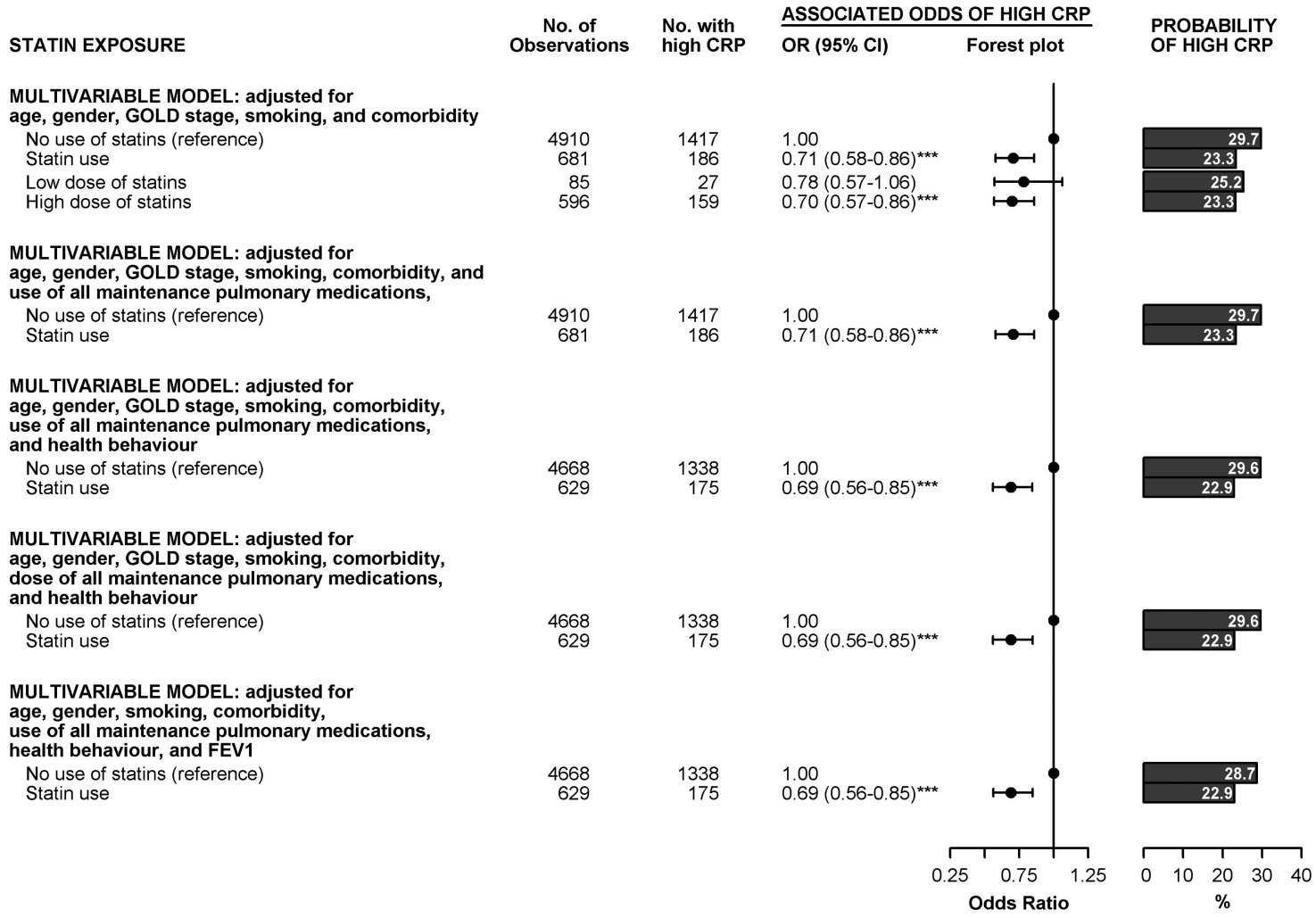

Figure 3 Statin use and C reactive protein (CRP). Results of the multivariable logistic regression analysis. From left to right: number of individuals in study ('No. of Observations'), number of individuals with high CRP ('No. with high CRP'), OR of high CRP with 95\% Cls, a corresponding forest plot, and a bar plot showing the average predicted probability of having a high CRP. Significance codes: ' ${ }^{* * * \prime}$ for $p<0.001$. Individuals with missing values on confounders in multivariable models were not included in the analyses, explaining why the numbers reported in the multivariable models differ slightly.

In a sensitivity analysis, we analysed the risk of exacerbations starting follow-up from 6 months after CRP measurement. In this analysis, a high CRP remained associated with exacerbations, $\mathrm{HR}=1.67$ (1.41 to $2.02, \mathrm{p}<0.001)$ (data not shown).

\section{Percentage of excess risk mediated}

We estimated from bootstrapped resampled PERM (CRP)'s that approximately $14 \%(4-51 \%)$ of the association of statin use with exacerbations in COPD may be mediated through a reduction of CRP; importantly, causal inference cannot be drawn from this analysis. This is shown in figure 5, which also shows a summary of our results.

\section{DISCUSSION}

Statin use was associated with reduced odds of exacerbations in individuals with COPD from the general population, although this was not apparent in those with the most severe COPD and without cardiovascular comorbidity. Statins may thus only associate with reduced risk of exacerbations in patients with COPD with coexisting cardiovascular disease. Importantly, causal inference cannot be drawn from our observational analyses.

Mechanistically, studies have shown that statins have several effects besides a lowering of plasma cholesterol, including antiinflammatory effects. ${ }^{8} 9$ Furthermore, there is evidence of systemic inflammation in a proportion of individuals with COPD. ${ }^{53}$ Our study indicates that, if present, part of a potential beneficial effect of statin use on exacerbations may by explained by reduction of systemic inflammation, marked as reduction of CRP in individuals with COPD and cardiovascular comorbidity. ${ }^{18}$
Studies using pharmaceutical databases have previously indicated that statin use is associated with reduced odds of exacerbations in COPD. ${ }^{34} 35$ The most recent and well-quoted study showed that a current statin use could be associated with $40 \%$ reduced odds of exacerbations. ${ }^{12}$ Our study finds similar associations in a setting where we were able to include several possible confounders such as the level of CRP. As expected, we observed that statin use in individuals with COPD is strongly associated with lower values of CRP, as shown previously in individuals without COPD. ${ }^{10} 11$

The question is then, how the results of our study should be interpreted in the light of the recent large well-conducted and convincingly negative RCT of simvastatin on risk of COPD exacerbations. ${ }^{14}$ In contrast to our study, the randomised study excluded all patients with cardiovascular comorbidity. There is substantial evidence of the burden of cardiovascular comorbidity in COPD, ${ }^{36}$ and studies have also shown that cardiovascular comorbidity may be linked to increased systemic inflammation in COPD. ${ }^{6} 37$ In our study, cardiovascular comorbidity was present in a large proportion of individuals with COPD. Inflammation caused by presence of cardiovascular comorbidity in COPD could act synergistically with pulmonary inflammation, and thereby explain our findings. Indeed, our subgroup analysis in individuals without cardiovascular comorbidity as well as the findings from the recent RCT suggest that statins have no effect on COPD exacerbations in patients with the most severe COPD and without cardiovascular comorbidity, which we believe supports our hypothesis.

If a potential beneficial effect of statins on exacerbations would only apply among individuals with cardiovascular 


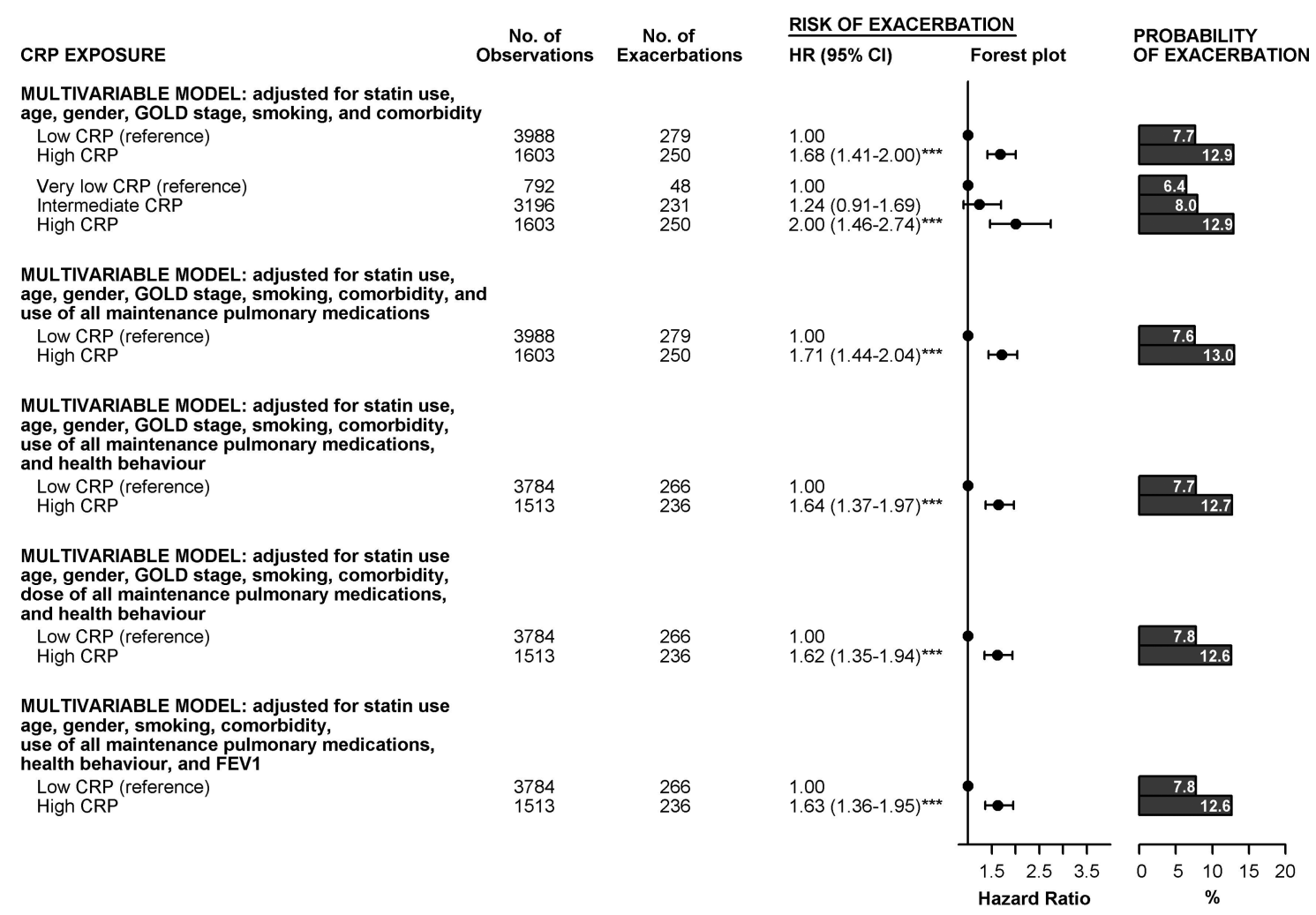

Figure $4 \mathrm{C}$ reactive protein (CRP) and exacerbations. Results of the multivariable Cox regression analysis. From left to right: number of individuals in study ('No. of Observations'), number of individuals with exacerbations ('No. of Exacerbations'), risk of exacerbations (HR) with $95 \%$ Cls, a corresponding forest plot, and a bar plot showing the average predicted probability of exacerbations during follow-up. Significance codes: ${ }^{* * * * \prime}$ for $\mathrm{p}<0.001$. Individuals with missing values on confounders in multivariable models were not included in the analyses, explaining why the numbers reported in the multivariable models differ slightly.

comorbidity, this would question the suggestion for giving statins to any patients with COPD. It could namely be argued that most individuals with cardiovascular disease should already be treated with statins, and therefore automatically would receive such treatment. However, this is not always the case and our study suggests that in patients with COPD with cardiovascular comorbidity focus on statin treatment possibly is important, despite the recent negative randomised trial in patients with COPD without cardiovascular comorbidity. ${ }^{14}$ Furthermore, our observations could also relate to a possible phenotypical variability such as 'systemic COPD', ${ }^{18} 38$ and questions whether statins could be preventing dyspnoea in primary pulmonary events, or dyspnoea in cardiac events in those with cardiovascular comorbidity. ${ }^{15}$

Clinicians are increasingly faced with the task of weighing up evidence from RCTs against findings from pharmacoepidemiology. RCTs are the most prominent way to assess treatment effects, but the results of a single study should be interpreted cautiously. ${ }^{39}$ Fortunately, RCTs and observational studies mostly come to the same conclusions, and observational studies can be used synergistically to test the external validity and formulate hypotheses. ${ }^{40}$ However, when the results of a single RCT and observational studies do not concur, it is important to consider possible reasons for the discrepancy. ${ }^{40}$

Observational studies may be limited by selection bias caused by unmeasured confounders (see figure 1 and see online supplementary e-figure 4) that could cause unmeasured differences between two treatment groups. On the other hand, observational data can be very important for estimating effects in patients who are frequently excluded from clinical trials, such as those with comorbidities. When observational studies restricted to a subgroup with the same set of exclusion and/or inclusion criteria as a RCT show similar results, the results from all individuals in the observational study could still be important. A rationale for performing observational analyses is the ability to provide estimates of drug effectiveness in patients more like those observed in clinical practice than those enrolled in clinical trials, since this can allow suggestions of precisely which patients may benefit from treatment. ${ }^{40}$

A possible limitation to our study is that severity of COPD was defined by prebronchodilator spirometry. Therefore, although we excluded all individuals with self-reported asthma, we cannot exclude the possibility that some individuals with asthma may have been included in the analyses. A possible bias in our study is a health behaviour bias; that is, that a person who regularly uses any kind of medication, or is regularly provided with healthcare, has certain lifestyle characteristics that may reduce the risk of exacerbations. To minimise such potential bias, multivariable analyses where adjusted for health behaviour and these adjustments did not change our estimates. However, individuals with, for example, previous exacerbations could be more likely to be started on statins, thereby leading to confounding by indication, which an adjustment for a healthy user effect might not have captured fully. Nevertheless, the subgroup analysis among individuals with the most severe COPD with previous exacerbations showed a null association between statin use and exacerbations, and therefore we believe that confounding by indication is unlikely to explain our main findings. Although we had complete data on follow-up for all individuals, another 

Statin use and associated odds of exacerbations (shown in figure 2). Statin use and associated odds of having a high $C$ reactive protein (CRP) level (>3 mg/L) (shown in figure 3). High CRP and associated risk of exacerbations (shown in figure 4). Estimates of the percentage of excess risk of the association of statins use with exacerbations possibly mediated of excess risk mediated (PERM) (CRP),
Figure 5 Summary of all results. through reduction of CRP, percentage with $95 \%$ Cls.

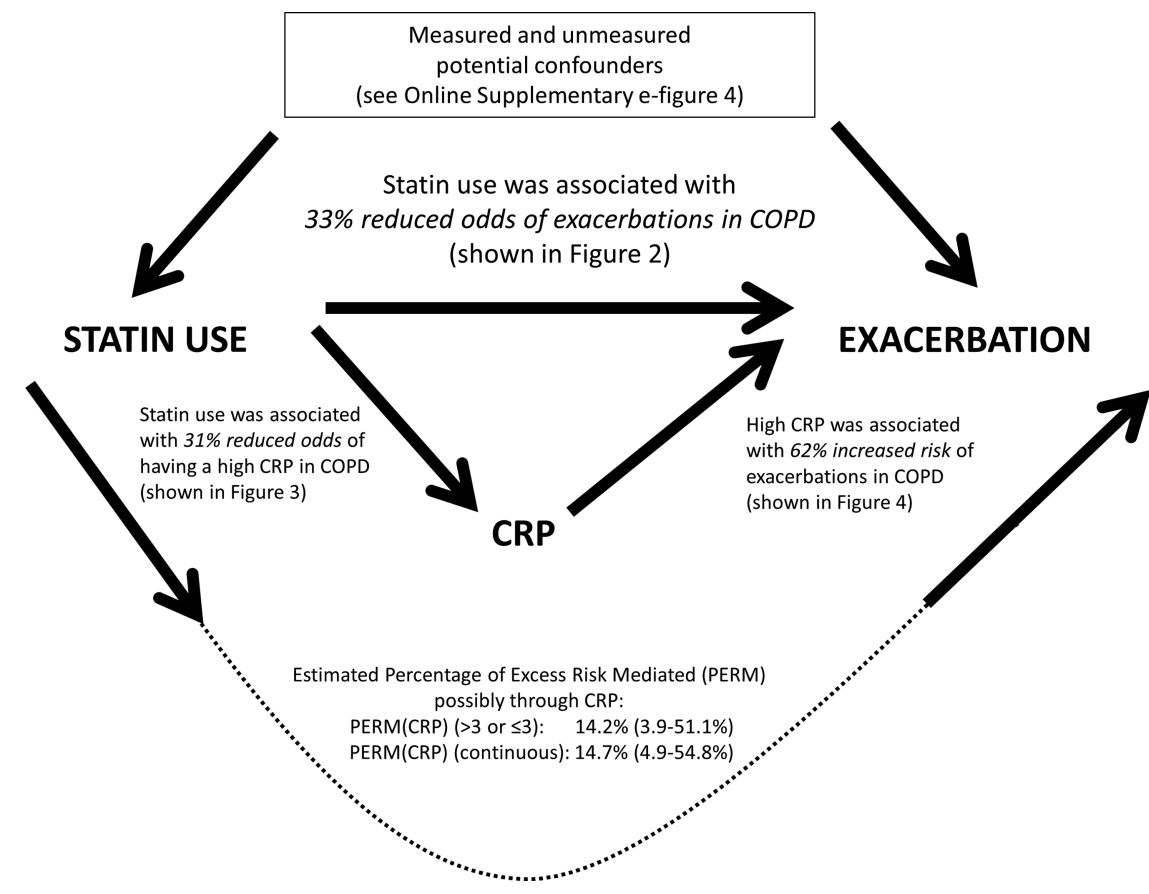

possible bias is missing baseline data, that could lead to selection bias. However, the percentages of missing values were low, and we consider selection bias as a result of this unlikely. Furthermore, as another possible limitation, even though we had information on all dispensing, that is, all physically retrieved medication at the pharmacy, this does not guarantee that the individuals actually took all of the medication.

Among the strengths of our study is the data collection at one site only with detailed information on all individuals, including pulmonary function tests, and complete follow-up with regard to exacerbations in all-inclusive nationwide registers. ${ }^{17} 19$ Furthermore, in our analysis, we were also able to adjust for use and dose of all maintenance pulmonary medications, that are expected to affect the risk of exacerbations, ${ }^{41} 42$ and our results where robust towards these adjustments. Further strengths of this study include the nested case-control design with a fixed lookback period, avoiding immortal time bias which can cause strong overestimation of treatment efficacy.

In conclusion, statin use was associated with reduced odds of exacerbations in individuals with COPD from the general population, although this was not apparent in those with the most severe COPD and without cardiovascular comorbidity. Statins may thus only associate with reduced risk of exacerbations in patients with COPD with coexisting cardiovascular disease. Importantly, causal inference cannot be drawn from these observational data.

\section{Author affiliations}

${ }^{1}$ Department of Respiratory Medicine, Odense University Hospital, and Institute of Clinical Research, University of Southern Denmark, Odense, Denmark

${ }^{2}$ The Copenhagen City Heart Study, Frederiksberg Hospital, Copenhagen, Denmark

${ }^{3}$ The Copenhagen General Population Study, Herlev Hospital, Copenhagen University Hospital, University of Copenhagen, Copenhagen, Denmark

${ }^{4}$ Department of Clinical Biochemistry, Herlev Hospital, Copenhagen University Hospital, University of Copenhagen, Copenhagen, Denmark

${ }^{5}$ Respiratory Section, Hvidovre Hospital, Copenhagen University Hospital, University of Copenhagen, Copenhagen, Denmark

${ }^{6}$ Department of Social Medicine, Institute of Public Health, University of Copenhagen, Copenhagen, Denmark

${ }^{7}$ Department of Clinical Pharmacology, University of Southern Denmark, Odense, Denmark

${ }^{8}$ Department of Respiratory Medicine, Gentofte Hospital, Copenhagen University Hospital, University of Copenhagen, Copenhagen, Denmark
${ }^{9}$ Respiratory and Allergy Research Group, Manchester Academic Health Sciences Centre, University Hospital South Manchester NHS Foundation Trust, Manchester, UK

Contributors Study concept and design: TSI, JV, BGN and JLM. Acquisition of data: revision of the manuscript: all authors. Statistical analysis: JLM, TSI, JV and BGN. Obtained funding: PL, JV and BGN. Study supervision: JV, BGN, JH, PL, TSI. TSI and JLM had full access to all the data in the study, and takes responsibility for the integrity of the data and the accuracy of the data analysis, and for the submission

Funding Supported by the Capital Region of Copenhagen, the Danish Heart Foundation, the Danish Lung Foundation, the Velux Foundation and Herlev Hospital.

Competing interests JV has received honoraria from GlaxoSmithKline, Almirall, AstraZeneca, Boehringer-Ingelheim, Novartis and Takeda for consulting and for presenting at meetings and symposia. PL has received honoraria from GlaxoSmithKline and other pharmaceutical companies for consulting, teaching and for presenting at meetings and symposia. JH has participated in research projects funded by Novartis, Pfizer, MSD, Nycomed and Alkabello with grants paid to the institution where he was employed, and has received fees for teaching or consulting from Nycomed, Pfizer, Novartis, Astra Zeneca and other pharmaceutical companies.

Ethics approval The study was approved by an institutional review board and the regional ethics committee (H-KF01-144/01), and was conducted according to the Declaration of Helsinki.

Provenance and peer review Not commissioned; externally peer reviewed.

\section{REFERENCES}

1 Lopez AD, Shibuya K, Rao C, et al. Chronic obstructive pulmonary disease: current burden and future projections. Eur Respir J 2006;27:397-412.

2 Pauwels RA, Rabe KF. Burden and clinical features of chronic obstructive pulmonary disease (COPD). Lancet 2004;364:613-20.

3 Hurst JR, Vestbo J, Anzueto A, et al. Susceptibility to exacerbation in chronic obstructive pulmonary disease. N Engl J Med 2010;363:1128-38.

4 Vestbo J, Hurd SS, Agusti AG, et al. Global strategy for the diagnosis, management, and prevention of chronic obstructive pulmonary disease: GOLD executive summary. Am J Respir Crit Care Med 2013;187:347-65.

5 Gan WQ, Man SF, Senthilselvan A, et al. Association between chronic obstructive pulmonary disease and systemic inflammation: a systematic review and a meta-analysis. Thorax 2004;59:574-80.

6 Sinden NJ, Stockley RA. Systemic inflammation and comorbidity in COPD: a result of 'overspill' of inflammatory mediators from the lungs? Review of the evidence. Thorax 2010;65:930-6.

7 Young RP, Hopkins R, Eaton TE. Potential benefits of statins on morbidity and mortality in chronic obstructive pulmonary disease: a review of the evidence. Postgrad Med J 2009;85:414-21.

8 Wang CY, Liu PY, Liao JK. Pleiotropic effects of statin therapy: molecular mechanisms and clinical results. Trends Mol Med 2008;14:37-44. PL and BGN. Analysis and interpretation of data: TSI, JLM, JV, BGN and JH. Critical 
9 Davignon J. Pleiotropic effects of pitavastatin. Br J Clin Pharmacol 2012;73:518-35.

10 Ridker PM, Danielson E, Fonseca FA, et al. Rosuvastatin to prevent vascular events in men and women with elevated C-reactive protein. $N$ Engl J Med 2008;359:2195-207.

11 Ridker PM, Danielson E, Fonseca FA, et al. Reduction in C-reactive protein and LDL cholesterol and cardiovascular event rates after initiation of rosuvastatin: a prospective study of the JUPITER trial. Lancet 2009:373:1175-82.

12 Wang MT, Lo YW, Tsai CL, et al. Statin use and risk of COPD exacerbation requiring hospitalization. Am J Med 2013;126:598-606.e2.

13 Janda S, Park K, FitzGerald JM, et al. Statins in COPD: a systematic review. Chest 2009;136:734-43.

14 Criner GJ, Connett JE, Aaron SD, et al. Simvastatin for the Prevention of Exacerbations in Moderate-to-Severe COPD. N Engl J Med 2014;370:2201-10.

15 Lange $\mathrm{P}$, Marott JL, Vestbo J, et al. Prediction of the clinical course of chronic obstructive pulmonary disease, using the new GOLD classification: a study of the general population. Am J Respir Crit Care Med 2012;186:975-81.

16 Ingebrigtsen TS, Marott JL, Vestbo J, et al. Characteristics of undertreatment in COPD in the general population. Chest 2013;144:1811-18.

17 Lynge E, Sandegaard JL, Rebolj M. The Danish National Patient Register. Scand J Public Health 2011;39(7 Suppl):30-3.

18 Thomsen $\mathrm{M}$, Ingebrigtsen TS, Marott JL, et al. Inflammatory biomarkers and exacerbations in chronic obstructive pulmonary disease. JAMA 2013;309:2353-61.

19 Kildemoes HW, Sørensen HT, Hallas J. The Danish National Prescription Registry. Scand J Public Health 2011;39:38-41.

20 WHO Collaborating Centre for Drug Statistics Methodology. [cited 9 August 2014]. http://www.whocc.no

21 Richardson DB. An incidence density sampling program for nested case-control analyses. Occup Environ Med 2004;61:e59.

22 D'Hoore W, Bouckaert A, Tilquin C. Practical considerations on the use of the Charlson comorbidity index with administrative data bases. J Clin Epidemiol 1996:49:1429-33.

23 Strom B. Pharmacoepidemiology. 4th edn. Chichester, UK: John Wiley \& Sons, 2005.

24 Essebag V, Platt RW, Abrahamowicz M, et al. Comparison of nested case-control and survival analysis methodologies for analysis of time-dependent exposure. BMC Med Res Methodol 2005;5:5.

25 Essebag V, Genest J Jr, Suissa S, et al. The nested case-control study in cardiology. Am Heart J 2003;146:581-90.

26 Lu Y, Hajifathalian K, Ezzati M, et al.; The Global Burden of Metabolic Risk Factors for Chronic Diseases Collaboration (BMI mediated effects). Metabolic mediators of the effects of body-mass index, overweight, and obesity on coronary heart disease and stroke: a pooled analysis of 97 prospective cohorts with 1.8 million participants. Lancet 2014;383:970-83.

27 The Comprehensive R Archive Network. [cited 9 August 2014]. http://cran.r-project. org/

28 Crawley M. The $R$ book. Imperial College London at Silwood Park. London, UK: John Wiley \& Sons, Ltd., 2007.

29 Lange $\mathrm{P}$, Marott JL, Vestbo J, et al. Socioeconomic status and prognosis of COPD in Denmark. COPD 2014;11:431-7.

30 Truelsen T, Gronbaek M, Schnohr P, et al. Intake of beer, wine, and spirits and risk of stroke : the copenhagen city heart study. Stroke 1998;29:2467-72.

31 Wium-Andersen MK, Orsted DD, Nielsen SF, et al. Elevated C-reactive protein levels, psychological distress, and depression in 73, 131 individuals. JAMA Psychiatry 2013:70:176-84

32 DiCiccio TJ, Efron B. Bootstrap confidence intervals. Stat Sci 1996;11:189-228.

33 Lahousse L, Loth DW, Joos GF, et al. Statins, systemic inflammation and risk of death in COPD: the Rotterdam study. Pulm Pharmacol Ther 2013;26:212-17.

34 Blamoun Al, Batty GN, DeBari VA, et al. Statins may reduce episodes of exacerbation and the requirement for intubation in patients with COPD: evidence from a retrospective cohort study. Int J Clin Pract 2008;62:1373-8.

35 Huang CC, Chan WL, Chen YC, et al. Statin use and hospitalization in patients with chronic obstructive pulmonary disease: a nationwide population-based cohort study in Taiwan. Clin Ther 2011:33:1365-70.

36 Sode BF, Dahl M, Nordestgaard BG. Myocardial infarction and other co-morbidities in patients with chronic obstructive pulmonary disease: a Danish nationwide study of 7.4 million individuals. Eur Heart J 2011;32:2365-75.

37 Sin DD, Man SF. Why are patients with chronic obstructive pulmonary disease at increased risk of cardiovascular diseases? The potential role of systemic inflammation in chronic obstructive pulmonary disease. Circulation 2003; 107:1514-19.

38 Garcia-Aymerich J, Gomez FP, Benet M, et al. Identification and prospective validation of clinically relevant chronic obstructive pulmonary disease (COPD) subtypes. Thorax 2011;66:430-7.

39 Concato J, Shah N, Horwitz RI. Randomized, controlled trials, observational studies, and the hierarchy of research designs. N Engl J Med 2000;342:1887-92.

40 Hannan EL. Randomized clinical trials and observational studies: guidelines for assessing respective strengths and limitations. JACC Cardiovasc Interv 2008;1:211-17.

41 Tashkin DP, Celli B, Senn S, et al. A 4-year trial of tiotropium in chronic obstructive pulmonary disease. N Engl J Med 2008;359:1543-54.

42 Calverley P, Pauwels R, Vestbo J, et al. Combined salmeterol and fluticasone in the treatment of chronic obstructive pulmonary disease: a randomised controlled trial. Lancet 2003:361:449-56. 